\title{
Update on Imaging in Rheumatology - Recent Advances
}

\author{
King Kenneth Cheung and Margaret A. Hall-Craggs
}

King Kenneth Cheung BSc MBBS PhD FRCR is a Specialist Registrar in Radiology at University College London Hospital, UK. Competing interests: none.

Margaret A Hall-Craggs BA, MRCP, FRCR, MD is a Consultant Radiologist at University College London Hospital, UK and Professor in Medical Imaging, University College London, UK. Competing interests: none.

Department of Radiology, University College Hospitals NHS Trust, 235 Euston Road, London NW1 $2 \mathrm{BU}$

\begin{abstract}
Imaging plays a vital role in the diagnosis and management of rheumatological disorders.

Traditionally plain film radiography was widely used in arthropathies to assess periarticular bony changes, which often reflect established changes at the late stage of disease and thus have limited value in early diagnosis and disease monitoring.

Magnetic resonance imaging (MRI) has become an important imaging modality in rheumatological disorders due to its ability to assess both morphological as well as functional changes, and plays a significant role in early diagnosis, monitoring disease evolution, assessing treatment responses and prognostication. More recently, advances in hardware and novel imaging sequences have aided the development of new MRI techniques; for example, whole-body MRI (WB-MRI) is gaining popularity and allows assessment of the overall inflammatory status in arthritis, whilst quantitative MRI shows promise in allowing more objective evaluation and standardisation of imaging-based assessment of inflammatory arthritis.

The role of other emerging imaging techniques including high-resolution peripheral quantitative computed tomography (HR-pQCT) in early detection and monitoring of periarticular bone damage, fluorescence optical imaging (FOI) in visualisation of active inflammation, and molecular imaging in investigation of pathogenesis and disease evaluation on a cellular level will also be discussed.
\end{abstract}

\section{Keywords}

Arthritis; arthropathy; arthritides; rheumatoid; rheumatological; inflammatory; imaging; MRI; PET;

\section{Word count}

1241 excluding references and abstract 


\section{Introduction}

Plain radiography has in the past been the primary imaging tool for the investigation of arthropathies. Radiographic features of the various types of arthritis have been extensively described in the literature and previously in this journal. However, structural bony changes, such as sclerosis and erosions, seen on radiographs, occur late in the course of disease and early changes are not apparent. The sensitivity of plain films is low and disease activity cannot be adequately assessed. Pelvic and lumbar spine radiography incurs a relatively high dose of ionising radiation; for example, the dose of an AP radiograph of the pelvis is 35 times that of a chest X-ray, and should be avoided where possible, particularly in children and adolescents. Magnetic resonance imaging (MRI) and ultrasound scanning are being used increasingly for the early diagnosis of arthropathies, and this facilitates early treatment that may prevent progression to irreversible structural damage.

In this article we describe recent advances in the imaging of rheumatological diseases. For a more in-depth review of the role of ultrasound, the interested reader is referred to the dedicated ultrasound chapter in this publication.

\section{Magnetic resonance imaging}

MRI has emerged as the most sensitive imaging modality for detecting arthritis and discriminating between acute and chronic inflammation. In recent years, substantial technical advances have been made in software and hardware, such as phased array coils, higher performance gradients, parallel imaging and advanced post-processing techniques. These advances have led to reduction in scan times and improved image quality. Other advances include isotropic 3D-imaging techniques, which reduce partial volume artefact and allow reformatting in multiple planes for improved visualisation of anatomy and pathology. High-field strength systems (e.g. 3T MRI) are increasingly being used in clinical practice, providing increased spatial resolution and signal-to-noise ratio.

\section{Applications of morphological MRI and common findings}

Conventionally, morphological and contrast-enhanced MRI are used to assess inflammatory arthritis. In general, short tau inversion recovery (STIR) and contrast-enhanced T1-weighted images are useful for assessing active inflammation.

In rheumatoid arthritis (RA) the diseased joints show proliferative synovitis on MRI. This inflammation leads to bone erosions, which in the acute phase demonstrate loss of cortical definition and presence of subcortical bone marrow oedema (osteitis). These are seen as high signal on STIR (which is fluid-sensitive) and contrast-enhanced images. Mature erosions show sharply marginated areas of trabecular bone loss with cortical defects. Tenosynovitis, a common finding, is seen as synovial sheath thickening with marked enhancement on post-contrast images.

In axial and peripheral spondyloarthropathies, sacroiliitis is a common feature and is seen on MRI as subchondral bone marrow oedema, synovitis, capsulitis and enthesitis. In the new Assessment of Spondyloarthritis International Society (ASAS) criteria for axial spondyloarthritis ${ }^{1}$, the presence of 
subchondral bone marrow oedema is mandatory for the diagnosis of acute sacroiliitis. In chronic sacroiliitis, periarticular fat metaplasia, sclerosis and erosions are seen, followed by ankylosis in some patients. Active spondylitis is seen as facet and costovertebral joint synovitis, enthesitis of spinal ligaments and corner inflammatory lesions due to bone marrow oedema of vertebral entheses. Fatty deposition at vertebral corners (fatty Romanus lesions) is highly specific for axial spondyloarthropathy.

\section{New MR techniques and clinical applications}

Whole-body MRI (WB-MRI): in recent years WB-MRI has been employed for the assessment of rheumatological diseases with multifocal and systemic involvement. It can detect inflammation at multiple sites, some of which may be subclinical, thus allowing estimation of total disease load and activity. WB-MRI also facilitates visualisation of enthesitis at multiple sites (Figure 1). A standardised scoring system based on WB-MRI is under development. ${ }^{1}$

Quantitative MRI: Conventional analysis of MRI studies is subjective and relies on the experience and expertise of the reading radiologist. Quantitative methods provide objective evaluation and have the potential for automation and standardisation, and have been explored in randomised clinical trials to evaluated treatment response. ${ }^{3}$ Novel quantitative MRI techniques include diffusion-weighted imaging (DWI), dynamic contrast enhancement (DCE) and chemical shiftencoded (CSE) MRI.

DWI-MRI measures the mobility of free water in living tissue, where areas of active inflammation have increased free water content that results in increased diffusivity. Apparent diffusion coefficient (ADC) values in these regions are higher than in non-inflamed tissue (Figure 2), and have been used to evaluate sacroiliitis and as a biomarker for measuring treatment response. ${ }^{4}$

DCE-MRI utilises serial imaging at short intervals (typically few seconds) during intravenous contrast administration. The dynamic enhancement profiles (time vs signal intensity) of the diseased region differ to normal tissue due to presence of inflammation and neoangiogenesis, and have been shown to correlate with disease activity and predict progression of erosive disease. ${ }^{5}$ It is also possible to derive quantitative physiological parameters by fitting dynamic enhancement data to pharmacokinetic models, which reflect exchanges between plasma and extracellular extravascular space. Such absolute parameters have the advantage of being independent of MRI hardware and parameters, and have shown promises in detecting synovial inflammation in early arthritis and assessment of tissue microcirculation. 0

CSE-MRI utilises the small difference between the precession frequencies of water and fat protons when exposure to a strong external magnetic field (i.e. inside an MRI scanner). This phenomenon allows quantitative measurement of water and fat composition, and by the same token bone marrow oedema and fat metaplasia, which reflect active inflammation and structural damage respectively in inflammatory arthritis (Figure 3). This technique has been successfully applied in a cohort of adolescent patients with sacroiliitis to quantify bone inflammation and healing. ${ }^{7}$ 


\section{Other novel imaging modalities}

Outside MRI, a number of novel imaging modalities for evaluating rheumatological disorders have emerged in recent years; these include:

Fluorescence optical imaging (FOI): This technique involves intravenous administration of a nonspecific fluorophor (typically indocyanine green, ICG), which is excited by light at the dark red spectrum. The emitting fluorescence is detected by an optical camera sensor and imaged serially every second over a few minutes. Active inflammation is seen as increased fluorescence intensity due to increased neovascularity. FOI allows quick overview of active inflammation and has the potential to detect subclinical inflammation that is not apparent to clinical examination or ultrasound. Unlike ultrasound, it has the advantage of being operator-independent, but is currently limited to evaluation of the hand and wrist, and does not provide morphological information. ${ }^{8}$

High-resolution peripheral quantitative computed tomography (HR-pQCT): HR-pQCT offers extremely high-resolution 3D imaging (typically $82 \mu \mathrm{m}$ isotropic voxel size) of bone structure with low radiation dose. The high-resolution and quantitative nature of this technique enables assessment of periarticular bone marrow density and cortical/ trabecular microarchitecture for detecting early erosive damage. ${ }^{9}$

Molecular imaging: The imaging modalities discussed so far - albeit with great promises and advances - rely on the detection of anatomical and structural changes related to the disease, and are insensitive to the preceding molecular and cellular changes in the very early stages of pathogenesis. Molecular imaging is a collective term used to describe techniques that employ molecular probes to allow visualisation of the underlying biochemical processes driving the disease. Probes are designed to target various disease processes such as inflammation, T/B-cells activation, activated macrophages, activated vascular endothelium and apoptosis. To enable visualisation of these processes, probes are bound to imaging tracers (typically radiolabelled which facilitate detection through tissue penetration) that are detected using modalities such as positronemission tomography CT (PET-CT) and single photon emission CT (SPECT). Molecular imaging has also shown great potential in the development of personalised therapy; for example, the use radiolabelled biologicals such as anti-TNF $\alpha$ and anti-CD20 allows confirmation of presence of these targets in a patient, which may aid in predicting and selecting the most efficacious treatment. ${ }^{10}$

\section{Acknowledgement}

This work was undertaken at UCLH/UCL, which receives funding from the Department of Health's NIHR Biomedical Research Centre funding scheme. The views expressed in this publication are those of the authors and not necessarily those of the UK Department of Health. 


\section{Key points}

- Structural bony changes seen on radiographs reflect late disease, and thus have limited role in early diagnosis and disease monitoring.

- MRI is highly sensitive for detecting early bony changes and synovial inflammation, and is becoming more routinely used for early diagnosis, disease/ treatment monitoring and prognostication of arthropathies,

- Novel MR techniques provide new avenue for disease evaluation: WB-MRI facilitates detection of inflammation at multiple sites; DWI-, DCE- and CSE-MRI provide quantitative and objective assessment of periarticular damage and may aid in standardisation and automation.

- Other developments include fluorescence optical imaging which provides a quick and noninvasive overview of active inflammation of extremities, and receptor-specific molecular imaging shows potential for novel monitoring of pathogenesis, disease evolution and treatment selection/response.

\section{Key references}

1. Sieper J, Rudwaleit M, Baraliakos X, et al. The Assessment of SpondyloArthritis international Society (ASAS) handbook: a guide to assess spondyloarthritis. Ann Rheum Dis. 2009;68 Suppl 2:ii1-44.

2. Østergaard M, Eshed I, Althoff CE, et al. Whole-body Magnetic Resonance Imaging in Inflammatory Arthritis: Systematic Literature Review and First Steps Toward Standardization and an OMERACT Scoring System. J Rheumatol. 2017; [epub ahead of print]

3. Conaghan PG, Østergaard M, Bowes MA, et al. Comparing the effects of tofacitinib, methotrexate and the combination, on bone marrow oedema, synovitis and bone erosion in methotrexatenaive, early active rheumatoid arthritis: results of an exploratory randomised MRI study incorporating semiquantitative and quantitative techniques. Ann Rheum Dis. 2016;75(6):102433.

4. Bray TJP, Vendhan K, Ambrose N, et al. Diffusion-weighted imaging is a sensitive biomarker of response to biologic therapy in enthesitis-related arthritis. Rheumatology (Oxford). 2017;56(3):399-407.

5. Huang J, Stewart N, Crabbe J, et al. A 1-year follow-up study of dynamic magnetic resonance imaging in early rheumatoid arthritis reveals synovitis to be increased in shared epitopepositive patients and predictive of erosions at 1 year. Rheumatology (Oxford). 2000;39(4):40716.

\section{Further Reading}

6. Maijer KI, Van der leij C, De hair MJ, et al. Dynamic Contrast-Enhanced Magnetic Resonance Imaging Using Pharmacokinetic Modeling: Initial Experience in Patients With Early Arthritis. Arthritis Rheumatol. 2016;68(3):587-96.10 Ai F, Ai T, Li X, Hu D, Zhang W, Morelli JN. Value of 
diffusion-weighted magnetic resonance imaging in early diagnosis of ankylosing spondylitis. Rheumatol Int 2012; 32: 4005-13.

7. Bray TJP, Bainbridge A, Punwani S, Ioannou Y, Hall-Craggs MA. Simultaneous Quantification of Bone Edema/Adiposity and Structure in Inflamed Bone Using Chemical Shift-Encoded MRI in Spondyloarthritis. Magn Reson Med. 2017; [epub ahead of print]

8. Klein A, Just GW, Werner SG, et al. Fluorescence optical imaging and musculoskeletal ultrasonography in juvenile idiopathic polyarticular disease before and during antirheumatic treatment - a multicenter non-interventional diagnostic evaluation. Arthritis Res Ther. 2017;19(1):147.

9. Tam LS. The Role of High-resolution Peripheral Quantitative Computed Tomography as a Biomarker for Joint Damage in Inflammatory Arthritis. J Rheumatol. 2016;43(10):1911-1913.

10. Put S, Westhovens R, Lahoutte T, Matthys P. Molecular imaging of rheumatoid arthritis: emerging markers, tools, and techniques. Arthritis Res Ther. 2014;16(2):208. 


\section{Figures}

Figure 1

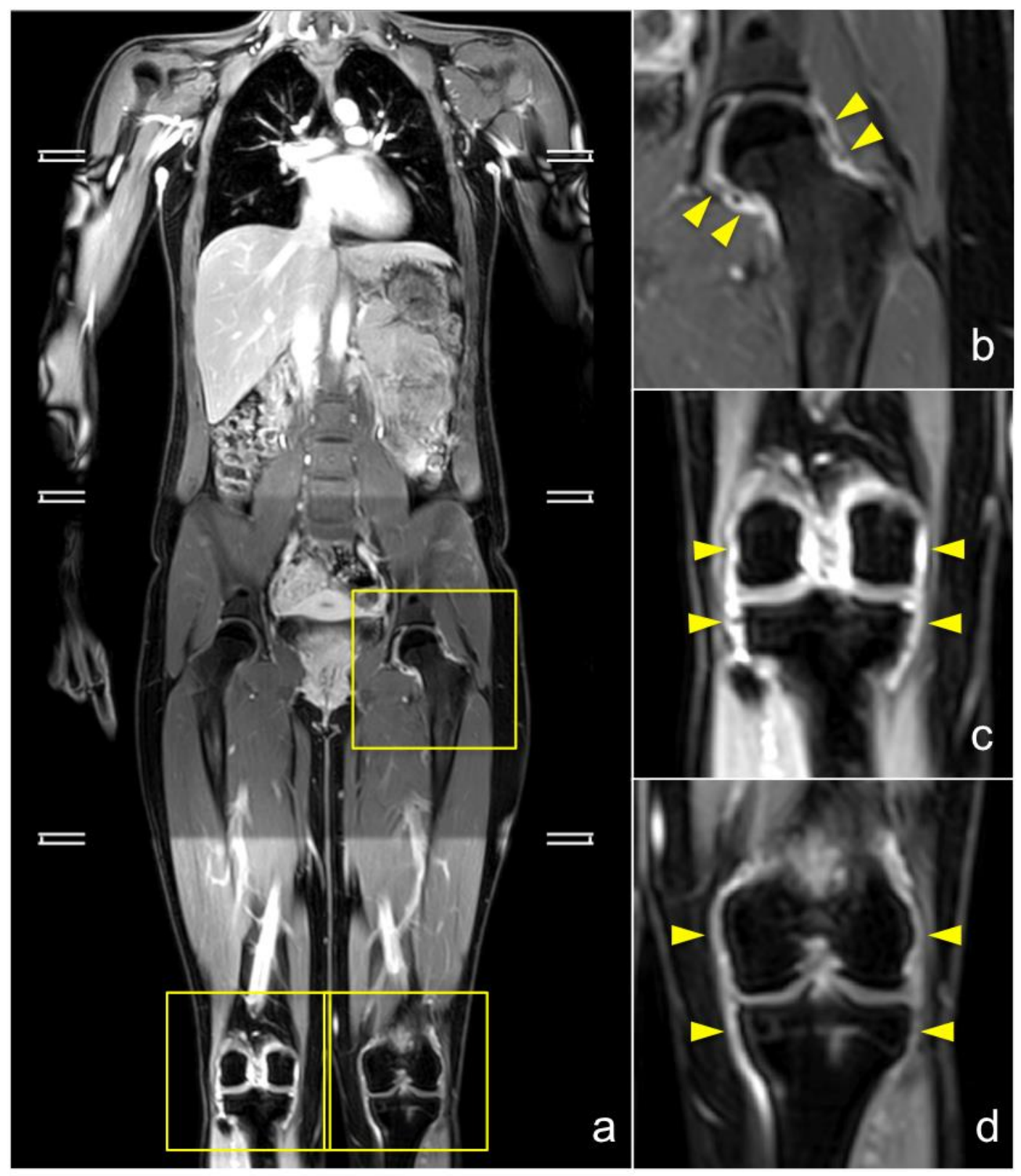

Figure 1: Whole Body MRI (WB-MRI) of a 22-year-old patient with polyarticular juvenile inflammatory arthritis. WB-MRI can image multiple joints facilitating the assessment of the distribution and severity of joint inflammation. (a) Large field-of-view image showing enhancing active synovitis in the left hip and right knee. Note the asymmetry of the hips with lack of synovial enhancement of the right hip. Magnified views of the diseased left hip (b) and right knee (c), [compare with normal left knee (d)] with arrowheads depicting the enhancing synovium. 
Figure 2

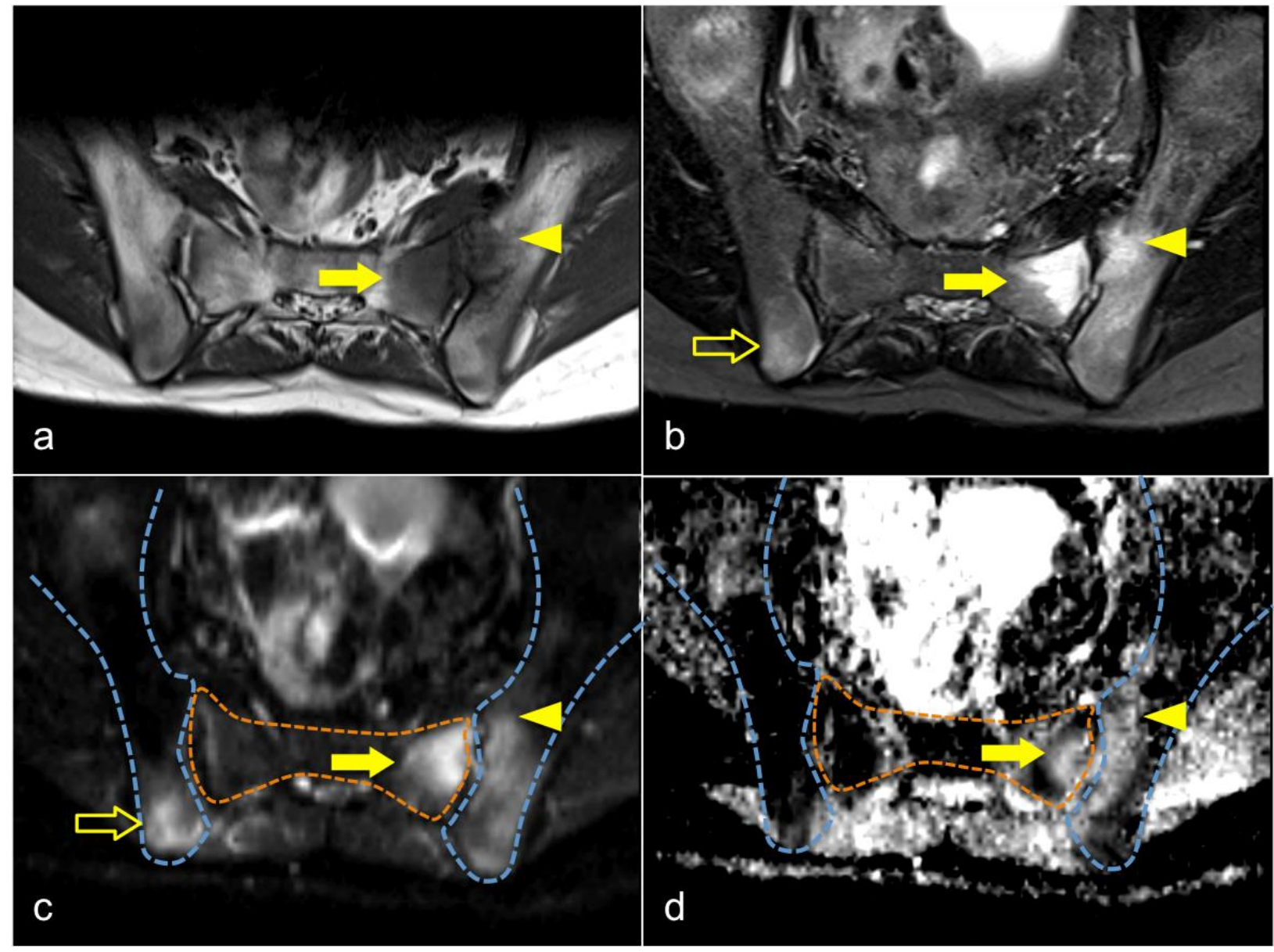

Figure 2: MRI of the sacroiliac joints of a 20 year-old patient with Enthesitis Related Arthritis and sacroiliitis. There is marked subchondral osseous oedema on the left joint, with less extensive involvement on the right. (a) T1-weighted image showing bone marrow oedema/ osteiits as low signal intensity, compared to the normal adjacent fatty marrow which is high signal on T1. (b) STIR, fluid sensitive sequence, demonstrating the corresponding foci of osteiitis as high signal regions. (c) Diffusion-weighted image (DWI) and (d) apparent diffusion coefficient (ADC) map. Areas of active osteiitis are hyperintense on both DWI and ADC images, reflecting unrestricted diffusion (arrowed c and d). Blue line - outline of ilium; Orange line - outline of sacrum.

\section{Figure 3}

Figure 3: Chemical-shift encoding (CSE) MRI. (TBC) 


\section{Self-assessment questions}

1. In inflammatory arthritis, which conventional MRI sequence is the most sensitive and specific for detection of active synovitis?
a. T1
b. Contrast-enhanced $\mathrm{T} 1$
c. T2
d. Proton density (PD)
e. STIR

2. An adolescent is referred to the rheumatology clinic for suspected polyarticular juvenile idiopathic arthritis. Standard blood test including antibody screening has been performed. The clinician is interested in ascertaining the distribution and extent of joint involvement. Which of the following imaging modality would be the most appropriate?
a. Radiography (X-ray)
b. Ultrasound
c. Molecular imaging with SPECT
d. Fluorescence optical imaging (FOI)
e. Whole-body MRI (WB-MRI)

3. The main potential advantage of molecular imaging over other imaging techniques in the evaluation of rheumatological disorders is its:
a. Lack of ionising radiation
b. Ability to target pathogenetic molecular pathways
c. Ability to assess structural changes
d. Low cost and availability
e. Non-invasive nature

\section{Answers to self-assessment questions}

1. Answer: (b). Out of the available options, contrast-enhanced T1 sequence is the most sensitive and specific for detecting active synovitis, where the increased neovascularity in the activelyinflamed synovium results in increased contrast-enhancement. Standard T1 sequence is insensitive to such change, but has a role in detecting bone marrow oedema (which is seen as hypointensity compared to adjacent normal bone marrow). There are often concurrent periarticular soft tissue oedema and joint fluid surrounding the site of active synovitis, to which T2, PD and STIR sequences are sensitive to in varying degrees, but these are secondary signs and are not as sensitive and specific compared to contrast-enhanced T1 sequence.

2. Answer: (e). WB-MRI, where available, is the most suitable option as it does not involve ionising radiation (which should be a major consideration in imaging children and adolescents due to increased risk of cancer), can image all joints in a single sitting to provide an overview of disease distribution, and allow evaluation of the deep joints of the axial skeleton. Radiography 
would not be the first choice as the radiation dose of imaging all joints would be prohibitive. Ultrasound offers a quick and readily accessible alternative, but is limited by its inability to visualise deep joints, and the time needed to image all joints would also be prohibitive. Molecular imaging with SPECT also involves a significant ionising radiation dose, is currently expensive and is not widely available. Fluorescence optimal imaging is currently limited to the hand and wrist, and is not yet widely available.

3. Answer: (b). The uniqueness of molecular imaging lies in its ability to target specific molecular and physiological processes that drive the disease, and has the potential to offer further insight into the pathogenesis of rheumatological disorders and personalisation of therapy. Most of the molecular probes being developed are bound to radiolabelled tracers such as technetium-99m, which are then detected using SPECT, thus this technique often involves ionising radiation. It is considered developmental and is not in clinical routine use, and production cost remains high. All of the imaging modalities discussed in this article are considered non-invasive, and involves no more than insertion of a standard cannula for the delivery of contrast agent/ tracer. 